\title{
When a Glue Sniffer Turns Weak
}

\section{Poh Juliana, Puneet Seth}

Department of Emergency Medicine, Singapore General Hospital, Singapore, Singapore.

Email: juliana.poh@sgh.com.sg

Received October $10^{\text {th }}, 2012$; revised November $30^{\text {th }}, 2012$; accepted December $11^{\text {th }}, 2012$

\begin{abstract}
Toluene inhalation can result in electrolyte and acid-base derangements and should be considered in the differential diagnosis of young patients with unexplained hypokalaemia and normal anion gap metabolic acidosis. This case serves to illustrate the abnormalities and heighten awareness among emergency physicians who may not have laboratory results on hand when evaluating causes of limb weakness.
\end{abstract}

Keywords: Toluene; Hypokalaemia; Renal Tubular Acidosis

\section{Introduction}

Glue sniffing or "toluene abuse" is becoming less common in the modern urban society and young emergency physicians may not always consider toxicological causes in patients presenting with limb weakness. We present a case of a chronic glue sniffer who presented with hypokalaemic periodic paralysis.

\section{Case}

A 42-year-old man was brought to the emergency department by ambulance after a fall at home, during which he hit his occiput against the wall. His main complaint was of generalized weakness for 4 days prior, with swallowing difficulty and shortness of breath. The weakness affected his legs before his arms. He denied having headache or pain in the neck, chest, abdomen or back. There was also difficulty controlling micturition and bowel movements. Sensation was intact. He gave a history of previous intravenous drug abuse and chronic glue sniffing and his last use was two days prior.

On examination, he was alert and had no abnormal odour. Blood pressure was $132 / 44 \mathrm{mmHg}$, heart rate $44 / \mathrm{min}$, respiratory rate $16 / \mathrm{min}$, and he was afebrile. There was no neck or back tenderness, no facial weakness, no paradoxical chest movements, no sensory deficits in limbs and trunk. Power in the upper and lower limbs was 2 out of 5 with decreased tone. He was areflexic in the upper limbs but knee jerks and ankle jerks were hyper-reflexic. Plantar reflexes were equivocal. Proprioception was intact. Anal tone was normal. A 12-lead ECG showed 2:1 heart block (Figure 1) and the patient was monitored in the resuscitation room.
Cervical spine and chest X-ray were normal. Bedside capillary blood sugar was $8 \mathrm{mmol} / \mathrm{L}$.

\begin{tabular}{cc}
\hline $\mathrm{ABG}$ (room air):pH & 7.27 \\
$\mathrm{PCO}_{2}$ & $27 \mathrm{mmHg}$ \\
$\mathrm{PO}_{2}$ & $106 \mathrm{mmHg}$ \\
Base excess & -15 \\
$\mathrm{HCO}_{3}$ & $15 \mathrm{mmol} / \mathrm{L}$ \\
$\mathrm{SaO}_{2}$ & $97 \%$ \\
Serum K & $1.08 \mathrm{mmol} / \mathrm{L}$ \\
\hline
\end{tabular}

Arterial blood gases on room air showed metabolic acidosis. Intraveous potassium chloride replacement was started in the emergency department. The results of his laboratory tests were as follows:

\begin{tabular}{cc} 
Haemoglobin & $16.1 \mathrm{~g} / \mathrm{dL}$ \\
White blood cell & $19.8 \times 10(9) / \mathrm{L}$ \\
Platelets & $316 \times 10(9) / \mathrm{L}$ \\
Urea & $1.8 \mathrm{mmol} / \mathrm{L}$ \\
Serum $\mathrm{K}^{+}$ & $<1.5 \mathrm{mmol} / \mathrm{L}$ \\
Sodium & $136 \mathrm{mmol} / \mathrm{L}$ \\
Chloride & $113 \mathrm{mmol} / \mathrm{L}$ \\
Bicarbonate & $10.8 \mathrm{mmol} / \mathrm{L}$ \\
Creatinine & $73 \mathrm{umol} / \mathrm{L}$ \\
Myoglobin & $249 \mathrm{uG} / \mathrm{L}$ \\
Free thyroxine & $5.5 \mathrm{pmol} / \mathrm{L}$ \\
Capillary sugar & $8 \mathrm{mmol} / \mathrm{L}$ \\
Troponin T & $0.08 \mathrm{uG} / \mathrm{L}$ \\
Creatinine kinase-MB & $8.5 \mathrm{uG} / \mathrm{L}$ \\
Anion gap & 13 \\
Urine pH & 6.2 \\
\hline
\end{tabular}




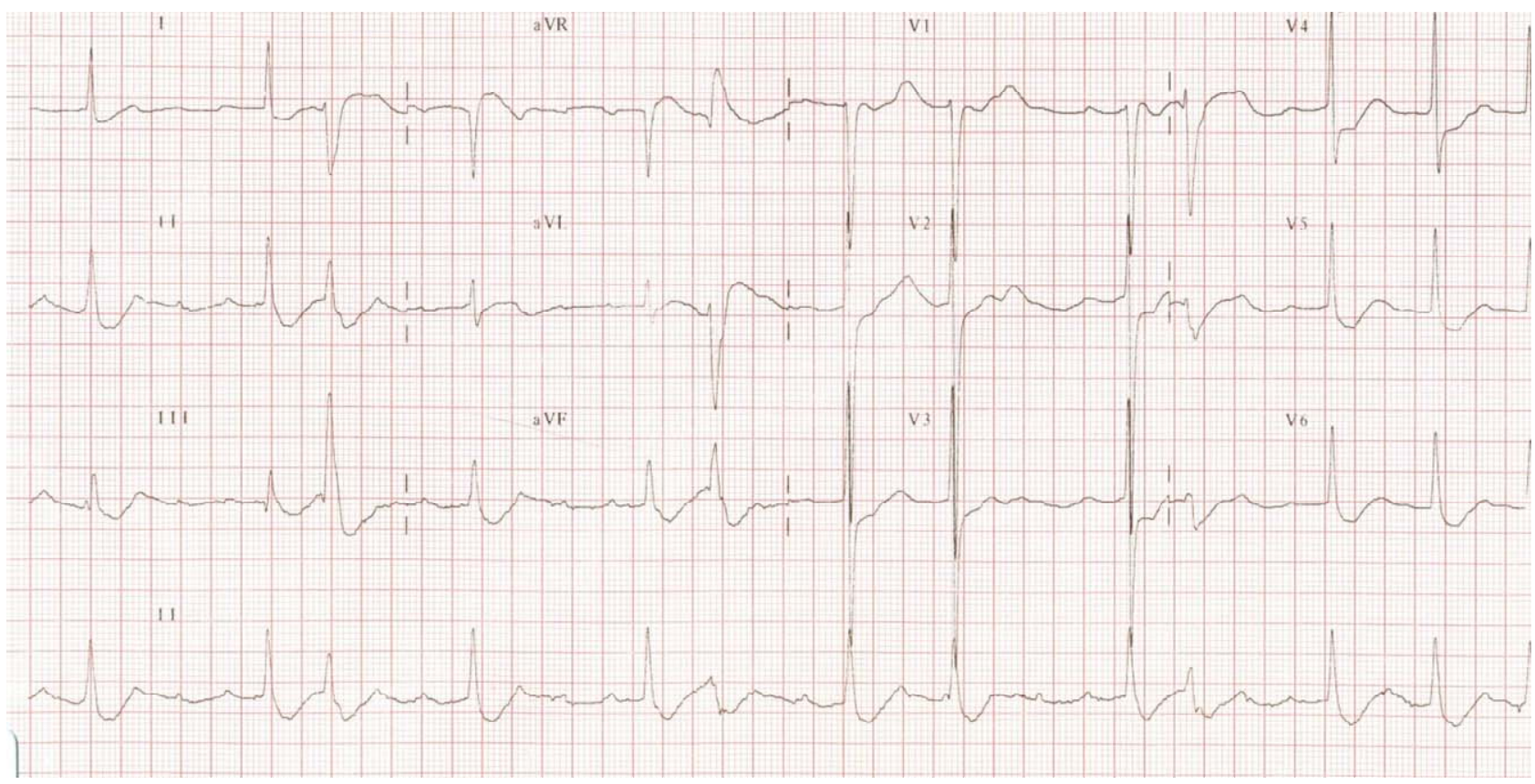

Figure 1. ECG showing 2:1 heart block.

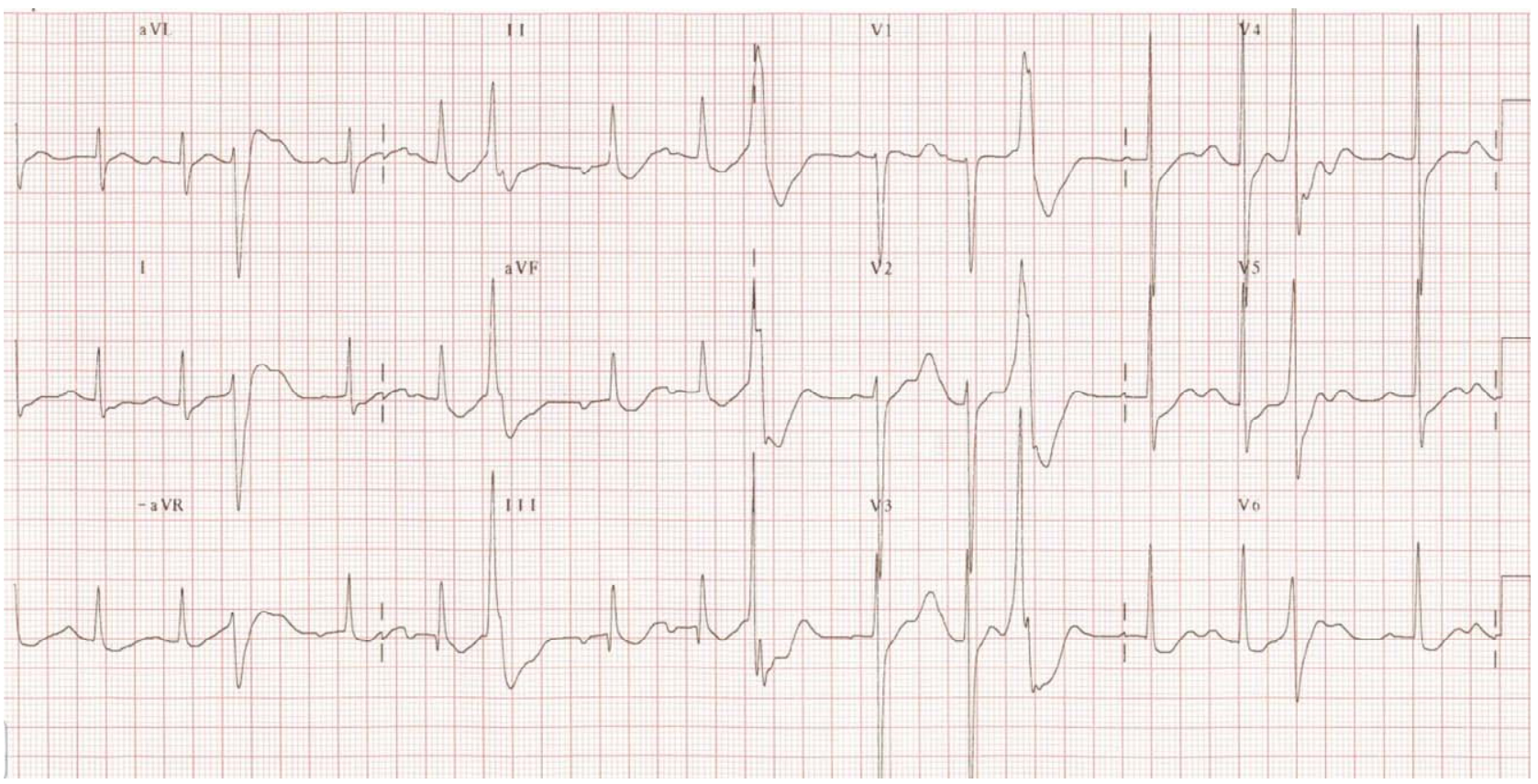

Figure 2. ECG showing premature atrial and ventricular complexes due to severe hypokalaemia.

He was diagnosed with hypokalaemic periodic paralysis and transferred to the medical intensive care unit for cardiac monitoring during aggressive potassium replacement in view of his arrhythmia (Figure 2). The patient required many cycles of $\mathrm{K}^{+}$replacement. He also had severe hypophosphataemia and hypocalcaemia with elevated magnesium levels and also rhabdomyolysis. Magnetic resonance imaging (cervico-thoracic spine) on Day 3 of admission showed only mild cervicalspondylosis and spastic paraparesis of his lower limbs was attributed to an accident many years prior. With restoration of normal potassium levels, the electrocardiogram reverted to sinus rhythm by Day 3 . There was full recovery of strength by Day 5 and he was discharged.

\section{Discussion}

Toluene is metabolized by the cytochrome P450 system, mainly in liver cytoplasm, and for every toluene metabo- 
lized, the formation of benzoic and hippuric acid adds one $\mathrm{H}+$ (i.e. acid load) to the body. Low net excretion of acid results in hyperchloraemic metabolic acidosis. However, the exact mechanism of toluene-induced distal renal tubular acidosis is still unknown and the severity of metabolic acidosis depends on various factors such as duration of toluene exposure, obesity and pre-existing renal disease.

It is interesting to note that when assessing patients with weakness secondary to hypokalaemic periodic paralysis, electrocardiographic abnormalities and an arterial blood gas reflecting metabolic acidosis with hypokalaemia will quickly alert the astute physician to the fact that the weakness is not neurological in nature. In patients with an unclear history of drug ingestion, toxicology screening would be helpful in establishing the diagnosis subsequently. The mainstay of treatment is cautious replacement of potassium by slow infusion with telemetry monitoring. Our patient made an uneventful recovery when potassium levels were normalized and his ECG became normal.

\section{Conclusion}

Toxicological causes of symmetrical neuromuscular weak- ness must always be considered, though they occur less often than neurological causes. Hypokalaemia can be due to a variety of medical conditions, such as distal renal tubular acidosis. Replacement of potassium must be done slowly and with close cardiac monitoring.

\section{REFERENCES}

[1] D. C. Batlle, S. Sabatini and N. A. Kurtzman, "On the Mechanism of Toluene-Induced Renal Tubular Acidosis," Nephron, Vol. 49, No. 3, 1998, pp. 210-218. doi: $10.1159 / 000185057$

[2] E. J. F. Carlisle, S. M. Donnelly, et al., "Glue-Sniffing and Distal Renal Tubular Acidosis: Sticking to the Facts," Journal of the American Society of Nephrology, Vol. 1, No. 8, 1991, pp. 1019-1027.

[3] J.-J Hong, J.-L Lin, et al., "Chronic Glue Sniffer with Hy perchloraemia Metabolic Acidosis, Rhabdomyolysis, Irreversible Quadriplegia, Central Pontine Myelinolysis and Hypothyroidism," Nephrology Dialysis Transplant, Vol. 11, No. 9, 1996, pp. 1848-1849. doi:10.1093/oxfordjournals.ndt.a027683

[4] R. H. Bennett, H. R. Forman, "Hypokalemic Periodic Paralysis in Chronic Toluene Exposure," Archives of Neurology, Vol. 37, No. 10, 1980, p. 673. doi:10.1001/archneur.1980.00500590097021 